inflammatory bowel disease (PSC-IBD); cumulative risk of $14 \%$ at 5 years and $17 \%$ at 10 years. SCENIC (2015) consensus guidelines recommend surveillance with chromoendoscopy (CE) and use of a high definition (HD) scope with white light colonoscopy. We aimed to assess the value of $\mathrm{CE}$ and $\mathrm{HD}$ scope in detection of dysplasia in PSC-IBD.

Methods We analysed all colonoscopic surveillance performed for PSC-IBD between 2010-2020 at Oxford University Hospitals ( $n=422$ procedures). Continuous variables were assessed by Kruskal-Wallis (three groups) and categorical variables by Chi-square test. Repeat measurements were accounted for by multilevel regression models with individual colonoscopies nested within patients. Multilevel ordinal logistic regression was used for assessment of bowel prep. Outcomes were adjusted for confounding variables (adj).

Results 359 colonoscopies were analysed in 91 patients with PSC-IBD (69/422 were excluded as prior dysplasia detected). $\mathrm{CE}$ and HD scope use increased in frequency over the 10year period $(p=0.02)$. HD were rarely used in those with bowel preparation deemed inadequate $(p<0.001)$, but there was no association between $\mathrm{CE}$ and bowel prep quality. Dysplasia detection (adenomatous and serrated lesions) was higher with CE compared with white light examination; $14 \%$ vs $3 \%$ $(p<0.001)$, and after adjusting for confounders $(p<0.009)$ and use of HD scope $(\mathrm{p}<0.01)$, OR 5.02 (CI 1.43-17.7). Dysplasia detection was higher with HD compared with standard definition (SD); $14 \%$ vs $6 \%(\mathrm{p}<0.04)$, but was non-significant after adjusting for confounders, OR 1.93 (CI 0.69-5.4). Dysplasia was more likely to be found on targeted than random colonic biopsies $(\mathrm{p}<0.001)$.

CE increased detection of serrated lesions (9\% vs $2 \%$ $(\mathrm{p}=0.06)$ OR 3.5 adj) and more so adenomatous dysplasia $(6 \%$ vs $1 \%(p=0.03)$ OR 12 adj) compared to white light. HD increased detection of serrated lesions (9\% vs $3.5 \%$ $(\mathrm{p}=0.04)$ OR $2 \mathrm{adj})$ but not adenomatous dysplasia (6\% vs $2.5 \%(p=0.19)$ compared to SD. (table 1$)$.

Conclusion CE is superior to $\mathrm{HD}$ and SD white light examination for dysplasia detection in PSC-IBD after accounting for confounders and performed better for adenomatous than serrated lesions.

\section{P056 TAPPING INTO ASCITES: SERVICE EVALUATION OF ELECTIVE DAY-CASE PARACENTESIS}

Austin See*, Joshua Cabada. University of Birmingham, Birmingham, UK

10.1136/gutjnl-2021-BASL.65

Background Liver disease has been on the rise since 2011, with 138.3 per 100,000 people being admitted to hospital with it as a primary diagnosis as of $2019 .{ }^{1}$ Ascites is the most common complication of liver cirrhosis. Large-volume paracentesis necessitates hospital admissions. ${ }^{2} 3$ Elective day-case paracentesis was introduced into the our trust in July 2019 to reduce inpatient bed occupancy. It was established under the condition of regular service review to ensure ongoing safety and cost-savings. This is a service evaluation following a 2019 review.

Objective To evaluate the complication rates and cost-savings of day-case paracentesis in patients with ascitic liver-disease.

Methodology Six months' cumulative data was collected retrospectively via the hospital's online clinical system for all day-case paracentesis between January and October 2020, excluding the coronavirus lockdown period. The data collected included: patient demographics, date and time of admission and discharge, volume drained, and complications. Cost-savings was based on avoidance of inpatient bed days per paracentesis compared to the 2017-18 average (3.5 days using inpatient paracentesis), cost per day-case and any readmission bed days within 7-days. Complications requiring hospital admissions or additional interventions were reviewed.

Results 9 patients with a total of 28 episodes of day-case paracentesis were included. The average length of stay was found to be 0.48 days. This is similar to the 2019 service evaluation (0.50 days) and less than the 3.5 days average of 2017-18 when inpatient paracentesis was used. 3 of 28 episodes were admitted with 2 requiring albumin cover overnight and 1 incidentally found to be anaemic and thrombocytopenic requiring transfusion. Therefore, 25 episodes of inpatient admissions were avoided. Using data from NHS Improvement, cost savings per episode of inpatient admission avoided was calculated to be $£ 1,899 .{ }^{4}$ Hence, over this 6 -month period, the total savings were $£ 1,899 \times 25$ episodes $=£ 47,475$. Decreased length of stay reduces admission costs and the risk of exposure to hospital-acquired infections and other potential complications. This planned and preemptive draining pathway enhances patient quality of life compared to going through A\&E triage. No significant complications were found.

Conclusion Day-case paracentesis at our trust resulted in substantially reduced healthcare costs compared to inpatient paracentesis without compromising patient safety.

\section{REFERENCES}

1. Liver disease profiles: statistical commentary, February 2020 [Internet]. GOV.UK 2020 [cited 2020 Oct 28]. Available from: https://www.gov.uk/government/publications/liver-disease-profiles-february-2020-update/liver-disease-profiles-statisticalcommentary-february-2020

2. When to suspect cirrhosis | Diagnosis | Cirrhosis | CKS | NICE [Internet]. [cited 2020 Oct 28]. Available from: https://cks.nice.org.uk/topics/cirrhosis/diagnosis/ when-to-suspect-cirrhosis/

3. Complications | Background information | Cirrhosis | CKS | NICE [Internet]. [cited 2020 Oct 28]. Available from: https://cks.nice.org.uk/topics/cirrhosis/backgroundinformation/complications/

4. NHS Improvement. Archived Reference Costs [Internet]. [cited 2021 Apr 11] Available from: https://webarchive.nationalarchives.gov.uk/20200501111106/ https://improvement.nhs.uk/resources/reference-costs/

\section{P057 LANREOTIDE FOR THE TREATMENT OF CHRONIC GASTROINTESTINAL BLEEDING FROM ANGIOECTASIAS IN PATIENTS WITH PORTAL HYPERTENSION: A CASE SERIES}

${ }^{1}$ Katrina Perkaska*, ${ }^{2}$ Yash Prasad, 'Louise Hogg, ${ }^{1}$ Audrey Dillon. ' Leeds Teaching Hospitals Trust, Leeds, UK; ${ }^{2}$ Countess of Chester Hospital, Chester, UK

\subsection{6/gutjnl-2021-BASL.66}

Background Gastrointestinal haemorrhage from angioectasia are a common feature of patients with portal hypertensive enteropathy. Patients with angioectasia continue to be a challenge to manage despite the advancement in endoscopy. Advanced liver disease, significant co-morbidities and older age are some of the reasons patients may be refractory to conventional treatment.

Somatostatin analogs have been used to good effect in acute bleeds in patients with portal hypertension but little data exists for its use in chronic gastrointestinal bleeding. Our 